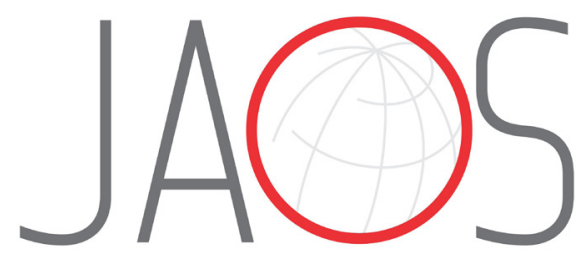

\title{
The effects of intro-oral parathyroid hormone on the healing of tooth extraction socket: an experimental study on hyperglycemic rats
}

\section{Abstract}

Lin $\mathrm{XU}^{1}$

$\mathrm{Li} \mathrm{MEI}^{2}$

Rui $\mathrm{ZHAO}^{3}$

Jianru $\mathrm{YI}^{1}$

Yixuan JIANG 4

Ruomei LI ${ }^{5}$

Youliang $\mathrm{ZHAO}^{6}$

$\mathrm{Li} \mathrm{PI}^{7}$

Yu LI ${ }^{1}$

Submitted: November 20, 2019

Modification: January 25, 2020

Accepted: February 24, 2020

Corresponding address: Professor Yu Li

State Key Laboratory of Oral Diseases - National Clinical Research Center for Oral Diseases Department of Orthodontics - West China Hospital of Stomatology - Sichuan University. No.14, South Renmin Road - section 3 Chengdu - 610041 - China. Phone: (+86) 028-85503645 e-mail: yuli@scu.edu.cn

Objective: To investigate the effects of intro-oral injection of parathyroid hormone (PTH) on tooth extraction wound healing in hyperglycemic rats. Methodology: 60 male Sprague-Dawley rats were randomly divided into the normal group $(n=30)$ and DM group $(n=30)$. Type 1 diabetes mellitus (DM) was induced by streptozotocin. After extracting the left first molar of all rats, each group was further divided into 3 subgroups ( $n=10$ per subgroup), receiving the administration of intermittent PTH, continuous PTH and saline (control), respectively. The intermittent-PTH group received intra-oral injection of PTH three times per week for two weeks. A thermosensitive controlled-release hydrogel was synthesized for continuous-PTH administration. The serum chemistry was determined to evaluate the systemic condition. All animals were sacrificed after 14 days. Micro-computed tomography (Micro-CT) and histological analyses were used to evaluate the healing of extraction sockets. Results: The level of serum glucose in the DM groups was significantly higher than that in the non-DM groups $(p<0.05)$; the level of serum calcium was similar in all groups ( $>0.05)$. Micro-CT analysis showed that the DM group had a significantly lower alveolar bone trabecular number (Tb.N) and higher trabecular separation (Tb.Sp) than the normal group $(p<0.05)$. The histological analyses showed that no significant difference in the amount of new bone (hard tissue) formation was found between the PTH and non-PTH groups ( $p>0.05)$. Conclusions: Bone formation in the extraction socket of the type 1 diabetic rats was reduced. PTH did not improve the healing of hard and soft tissues. The different PTH administration regimes (continuous vs. intermittent) had similar effect on tissue healing. These results demonstrated that the metabolic characteristics of the hyperglycemic rats produced a condition that was unable to respond to PTH treatment.

Keywords: Diabetes mellitus. Tooth extraction. Parathyroid hormone. Wound healing.

${ }^{1}$ State Key Laboratory of Oral Diseases, National Clinical Research Center for Oral Diseases, Department of Orthodontics, West China Hospital of Stomatology, Sichuan University, Chengdu, China.

${ }^{2}$ Discipline of Orthodontics, Department of Oral Sciences, Sir John Walsh Research Institute, Faculty of Dentistry, University of Otago, New Zealand.

${ }^{3}$ State Key Laboratory of Oral Diseases, National Clinical Research Center for Oral Diseases, Department of Maxillofacial Surgery, West China Hospital of Stomatology, Sichuan University, Chengdu, China.

${ }^{4}$ State Key Laboratory of Oral Diseases, National Clinical Research Center for Oral Diseases, Department of Oral Implantology, West China Hospital of Stomatology, Sichuan University, Chengdu, China.

${ }^{5}$ Department of Orthodontics, Ninth People's Hospital, School of Stomatology, Shanghai Key Laboratory of Stomatology, Shanghai Jiao Tong University, Shanghai, China

${ }^{6}$ Department of emergency department, West China Second Hospital, Sichuan University, Chengdu, China

${ }^{7}$ West China School of Public Health, Sichuan University, Chengdu, China 


\section{Introduction}

Diabetes mellitus (DM) has an increasingly higher occurrence of 463 million adults worldwide. ${ }^{1}$ People with DM are more likely to suffer from earlier detrimental oral status and are prone to numerous oral diseases, including oral infection, periodontitis and difficulties in wound healing after tooth extraction. ${ }^{2}$ It has been found that the prevalence of tooth extractions in DM patients is 1.88 times higher than the general population. ${ }^{3}$

The high-glucose microenvironment would delay the healing of tooth extraction sockets. ${ }^{4}$ High glucose could affect the osteoblastic function and matrix mineralization. ${ }^{5}$ The soft tissue repair is associated with the defect in the formation of granulation tissue and collagen degradation induced by excessive matrix metalloproteinase. ${ }^{6}$ The dysfunction of fibroblast could impair the collagen synthesis, resulting in decreased fiber accumulation. ${ }^{7}$ Many studies have explored different approaches to accelerate the healing of tooth extraction sockets under DM conditions, such as the use of growth factors, ${ }^{8}$ graft fillings, ${ }^{9}$ ellagic acid, ${ }^{10}$ and low-level laser therapy. ${ }^{11}$ The effectiveness of these approaches, however, is unsatisfactory due to the complexity of DM and vulnerability of the oral environment. ${ }^{12}$

Parathyroid hormone (PTH) is one of the important hormones to maintain the balance of the calcium and phosphorus metabolism. ${ }^{13} \mathrm{PTH}$ can regulate osteoclast activity and influence bone remodeling, and is currently the only clinical drug to promote osteogenesis for the treatment of osteoporosis. ${ }^{14}$ The intermittent administration of PTH has been found to directly inhibit transcription of the sclerosis gene and stimulate bone formation; ${ }^{15}$ the continuous administration of PTH could increase the receptor activator of the nuclear factor-kB ligand/osteoprotegerin ratio, resulting in an increased osteoclastogenesis. ${ }^{16}$ PTH could also benefit the healing of soft tissue via suppressing inflammation and stimulating collagen deposition. ${ }^{4} \mathrm{~A}$ recent study has reported that PTH promoted the healing of both hard and soft tissues in the tooth extraction socket. ${ }^{17}$

The effect of PTH on extraction socket healing under DM condition remains unclear. The aim of this study was to investigate and compare the effects of local administration (intro-oral injection) of intermittentand continuous-PTH on the healing of tooth extraction sockets in hyperglycemic rats.

\section{Methodology}

\section{Synthesis and characterization of PTH controlled-release hydrogel}

A thermosensitive and injectable hydrogel was prepared for the continuous administration of PTH based on the literature. ${ }^{18}$ The poly (ethylene glycol)-poly (caprolactone)-poly (ethylene glycol) thermosensitive hydrogel (PEG-PCL-PEG, PECE) was synthesized by ring-opening copolymerization. ${ }^{19}$ The ${ }^{1} \mathrm{H}$ Nuclear Magnetic Resonance ( ${ }^{1} \mathrm{H}$ NMR) spectra (in $\mathrm{CDCl}^{3}$ ) was recorded on a Varian 400 spectrometer (Varian, USA) at $400 \mathrm{MHz}$ to characterize chemical composition of PECE copolymers.

The sol-gel-sol phase transition behavior was carried out as follows: PECE hydrogel was placed into $5 \mathrm{~mL}$ EP tube and incubated in a water bath at $0^{\circ} \mathrm{C}$ for ten min, and then was slowly heated at a rate of $0.5^{\circ} \mathrm{C} / \mathrm{min}$ until $37^{\circ} \mathrm{C}$. The sol-gel-sol phase transition diagram was recorded using the test tube-inverting method, which was visually observed by inverting the tube. The condition of sol and gel phase was defined as "flow liquid sol" and "no flow solid gel".

In vitro release tests at body temperature were carried out as follows: firstly, $750 \mu \mathrm{L}$ hydrogel loaded with $1 \mathrm{mg}$ BAS protein was placed into the bottom of the EP tube at $37^{\circ} \mathrm{C}$ for $1 \mathrm{~h}$ to form the gel. Then, 1000 $\mu \mathrm{L}$ Milli-Q water was added into the tube to immerse the gel. Then, the sample was kept and shaken at 106 rpm at $37^{\circ} \mathrm{C}$. The solution was collected at each time of interest $(T=0,1,2,4,8,12,24 \mathrm{~h}$ in the first day, and at each $24 \mathrm{~h}$ interval until $336 \mathrm{~h}$ ) and replaced with 1000 $\mu \mathrm{L}$ preheated fresh Milli-Q water. After centrifugation at $13,000 \mathrm{rpm}$ for $10 \mathrm{~min}$, the measured extracts were stored at $-20^{\circ} \mathrm{C}$ for drug release analysis. The amount of released protein at each time points was assessed in triplicate using the Enhanced BCA Protein Assay kit (Beyotime technology, Beijing, China) following manufacturer's instructions. The accumulative release percent of BSA protein was calculated.

\section{Animal model and tooth extraction}

A total of 60 young male Sprague-Dawley rats (5 weeks old) were included in the study. Sample size was calculated by power analysis based on the estimated effect size reported in the previous study. ${ }^{20}$ Rats were housed at $22{ }^{\circ} \mathrm{C}, 40 \%$ humidity, and in 12-hour daylight cycles. Rats were allowed access to standard laboratory rodent diet and water. The ethics 
of the study was approved by the Research Ethics Committee of West China Hospital of Stomatology (No. 201610610379). The study was presented following the Animal Research N3CRs guidelines for Reporting of In Vivo Experiments (ARRIVE) guidelines.

Sixty rats were randomly divided into two groups ( $n=30$ per group), including the normal control and the diabetic group. Diabetes was induced into $12 \mathrm{~h}$-fasted rats by intraperitoneally streptozotocin (STZ) (Sigma, St Louis, MO, USA) dissolved in citrate buffer $(\mathrm{pH}=4.2-$ 4.5). STZ was calculated at $45 \mathrm{mg} / \mathrm{kg} .{ }^{21}$ Three days after the STZ injection, blood was obtained from the tail vein, and glucose levels were measured using a glucometer (Yuwell, Jiangsu, China). Diabetes was confirmed twice by the presence of random blood glucose concentrations of $>16.7 \mathrm{mmol} / \mathrm{L} .{ }^{10}$

The mandibular left first molar was extracted under general anesthesia (10\% chloral hydrate solution, $0.32 \mathrm{ml} / 100 \mathrm{~g}$ ) from each animal. Sterile dental instruments were used during the extraction of molars, and teeth removal was performed by using a sharpened dental explorer. ${ }^{22}$ Alveolar sockets were left to heal without sutures.

\section{Grouping and administration of PTH}

After tooth extraction, the two groups were divided randomly into 3 subgroups ( $n=10$ per group), including the normal saline (NS), the intermittent-PTH (iPTH) and the continuous-PTH group (CPTH).

The intermittent intra-oral injection of PTH (1-34) (Chinese Peptide, Hangzhou, China) was administered at a dose of $80 \mu \mathrm{g} / \mathrm{kg}$ three times a week for 14 days. ${ }^{17}$ ${ }^{23}$ It was dissolved in normal saline solution, and the needle was placed in the buccal vestibule next to the tooth extraction site.

The continuous intra-oral injection of PTH (134) was administered as follows: PECE was mixed and dissolved completely in NS to form sol in the temperature of $55-60^{\circ} \mathrm{C}$ at a concentration of 25 wt $\%$ and cooled at $0{ }^{\circ} \mathrm{C}$ for three minutes. ${ }^{19}$ Then, PTH (1-34) solution was mixed into the PECE sol to form suspension, and the PTH (1-34) gel to release slowly for 14 days was obtained. Soon after the tooth extraction, PECE hydrogel loaded with an equivalent dosage of PTH (1-34) in iPTH group was put in the tooth socket.

Animals in the two NS groups (NS normal group and NS diabetic group) were injected with the same volume of normal saline solution three times a week for 14 days.

\section{Serum chemistry}

Blood was collected from carotid artery at the time of euthanasia, which was $24 \mathrm{~h}$ after the last injection of PTH. Serum samples were prepared and used immediately. The levels of calcium were measured using a commercial kit (Jiancheng, Nanjing, China). The blood glucose levels from the tail vein of each group were monitored once every week using a glucometer (Yuwell, Jiangsu, China). Diabetic rats with unsatisfactory serum glucose levels would be removed; however, no animal was excluded for this reason.

\section{Micro computed tomography (Micro-CT) assessment}

Rats were euthanized at the end of the designated injection period of 14 days. The mandibles were dissected, and fixed in $10 \%$ formalin, and the extraction wounds were scanned by Micro-CT (MicroCT50, Scanco Medical, Bassersdorf, Switzerland) at $10 \mu \mathrm{m}$ voxel resolution with the beam energy level of $70 \mathrm{kVp}$ and electrical current of $0.2 \mathrm{~mA}$. The time of scanning was $858 \mathrm{~ms}$. Each mandible was fixed by upside-down styrofoam, and the occlusal plane became horizontal to the bottom. The conservation of the samples occured in $10 \%$ formalin. The thresholds were determined visually in several slices. Trabecular parameters were identified by the direct measurement technique. The region of interest was a trabecular compartment of 100 slices around extraction sockets and was segmented by semi-manual contour. Alveolar bone trabecular number (Tb.N), trabecular thickness (Tb.Th), trabecular separation (Tb.Sp) and bone mineral density (BMD) were analyzed with built-in software.

\section{Histomorphometric analysis}

Rats were decapitated two weeks after the interventions. The specimens were demineralized in $10 \%$ ethanol, paraffin-embedded, and sectioned to 5 $\mu \mathrm{m}$ thickness. Hematoxylin and eosin (HE) (Beyotime technology, Beijing, China) and Masson's trichrome (Sigma, St Louis, MO, USA) staining techniques were performed for the detection of bone tissue and collagen fibers respectively, following the manufacturer's instructions. The staining sections were analyzed by Image-Pro Plus (Media Cybernetics, Bethesda, MD, USA). New alveolar bone formation was quantified in 
the extraction wounds as described previously. ${ }^{20}$

\section{Statistics analysis}

Statistical analyses were performed using the SPSS statistics base 17 (SPSS Inc., Chicago, IL, USA). Results are presented as mean \pm standard deviation. The normality test was performed using the ShapiroWilk analysis; the statistical comparisons were analyzed by the Student's t-test, one-way ANOVA, and two-way ANOVA, with a significance level of $5 \%$. Statistical significance was defined as 0.05 a-level.

\section{Results}

\section{Characterization of PTH controlled-release hydrogel}

The chemical structure of PECE gel was characterized by ${ }^{1} \mathrm{H}$ NMR (Figure 1 ). The peak of the caprolactone unit and the ethylene glycol unit were at $3.98 \mathrm{ppm}$ and $3.57 \mathrm{ppm}$, respectively. The macromolecular weight estimated from ${ }^{1} \mathrm{H}$ NMR spectrum was 3246 , which was consistent with theoretical estimation, indicating that the PECE gel was synthesized successfully.

The temperature-dependent sol-gel phase transition behavior was presented in Figure $2 \mathrm{~A}$. The hydrogel showed a sol state at lower temperature $\left(0^{\circ} \mathrm{C}\right)$ and a gel state at body temperature $\left(37^{\circ} \mathrm{C}\right)$. The hydrogel underwent sol-gel-sol phase transition as the temperature increased and slowly released PTH at local part, which prolonged the therapeutic effect time and reduced the repeated injections.

The controlled-release ability of PECE hydrogel is shown in Figure 2B. The release rate of the drug was rapid in the first 168 hours, and then became comparatively slow and sustained in the next 168 hours. The cumulative release of protein reached $58.24 \%$ by the 14 th day. Therefore, the experiment period for the PTH controlled-release hydrogel was set as 14 days in this study.

\section{Serum chemistry}

The level of serum glucose in the DM groups $(\mathrm{DM}+\mathrm{NS}, \mathrm{DM}+\mathrm{iPTH}$ and $\mathrm{DM}+\mathrm{CPTH})$ (mean 22.38 \pm 2.71 $\mathrm{mmol} / \mathrm{L}$, with no significant difference within the three groups, $p>0.05$ ) was significantly higher than the nonDM groups (NOR+NS, NOR+iPTH and NOR+cPTH), (mean $5.82 \pm 0.67 \mathrm{mmol} / \mathrm{L})(\mathrm{p}<0.01)$, indicating that the DM model was successfully established in the study.

No statistical significance of the serum calcium concentration was found either between the DM and non-DM groups, or within these groups ( $p>0.05$ for all). Local administration in the extraction socket in the study could reduce the systematic effects of PTH, such as hypercalcemia, which was reflected by the unaltered serum calcium level.

\section{Micro-CT analysis of the extraction socket}

The Micro-CT analysis (Figure 3 ) showed that the Tb.N in NOR+NS group $\left(2.37 \pm 0.29 \mathrm{~mm}^{-1}\right)$ was significantly higher than that in DM+NS group (1.52 $\left.\pm 0.14 \mathrm{~mm}^{-1}\right)(\mathrm{p}<0.01)$ (Figure $\left.1 \mathrm{E}\right)$, while the Tb.Sp in NOR+NS group $(0.21 \pm 0.09 \mathrm{~mm})$ was significantly lower than that in DM+NS group $(0.47 \pm 0.05 \mathrm{~mm})$ $(p<0.05)$ (Figure $1 F)$, indicating that bone density was affected by the DM condition. No significant difference

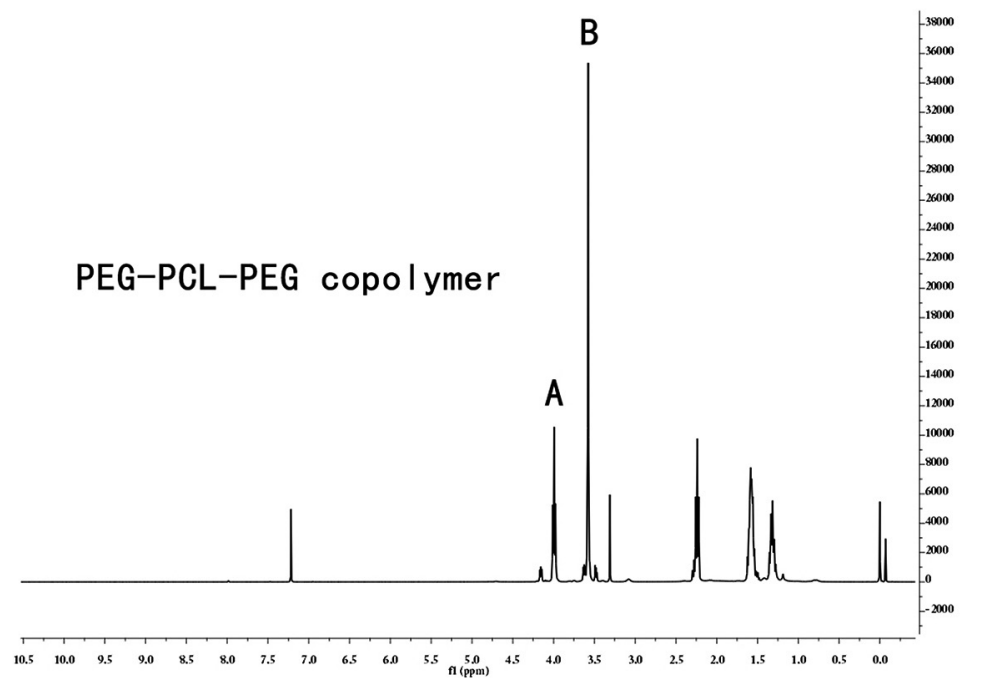

Figure 1- ${ }^{1} \mathrm{H}$ NMR spectrum of PECE copolymer. The peak of $(\mathrm{A})$ caprolactone unit and $(\mathrm{B})$ ethtlene glycol unit were at 3.98ppm and $3.57 \mathrm{ppm}$, respectively 


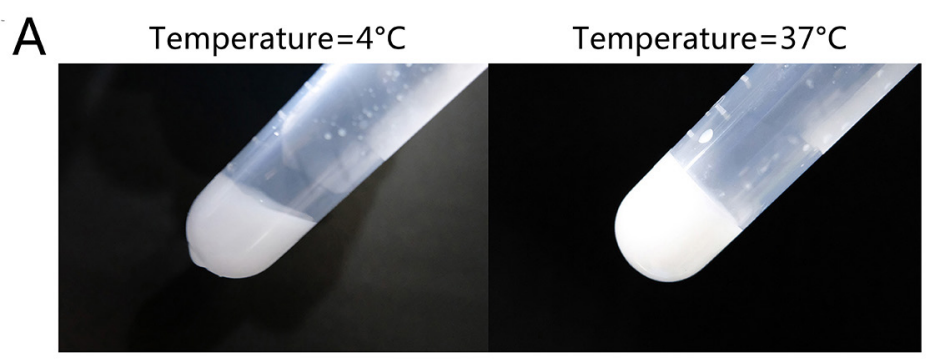

B

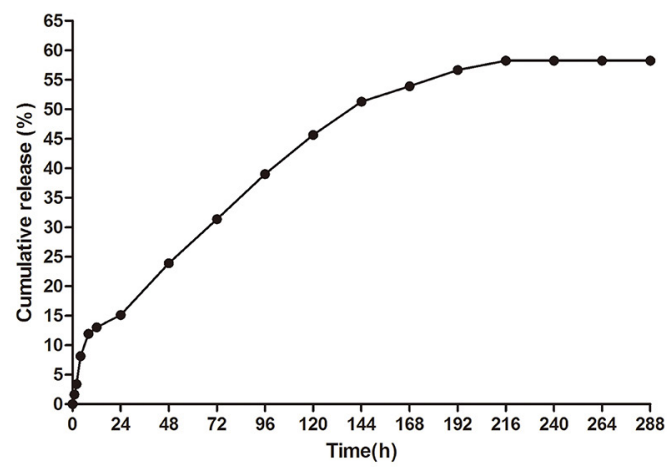

Figure 2- Characterization of PTH controlled-release hydrogel (A) The sol-gel phase transition behavior of PECE hydrogel: the solution state of hydrogel at $0^{\circ} \mathrm{C}$ and the gel state of hydrogel at $37^{\circ} \mathrm{C}$. (B) The cumulative release of drug-loaded PECE hydrogel for 14 days

A

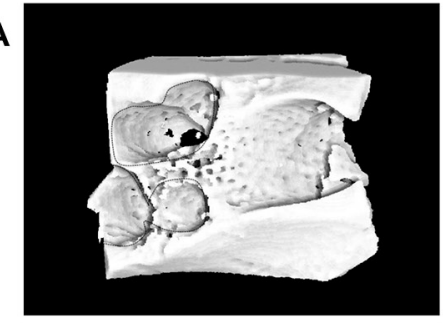

C

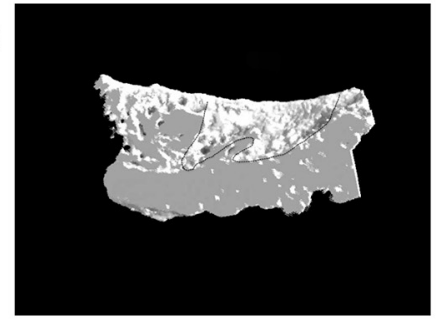

E
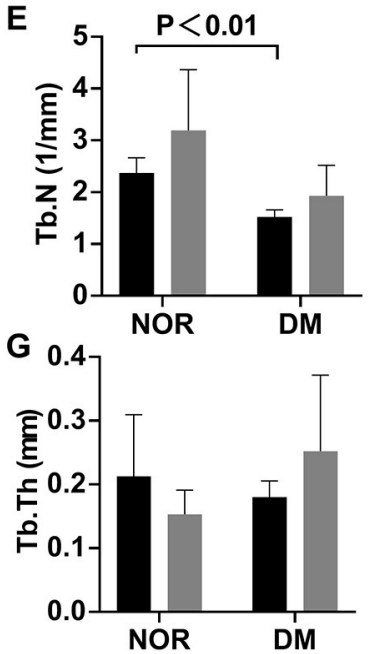

B

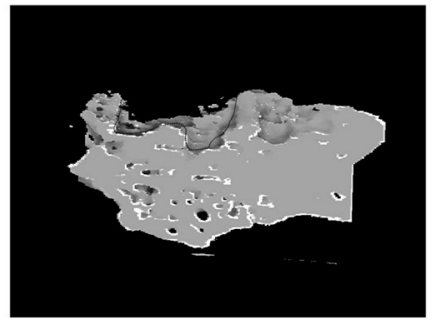

D

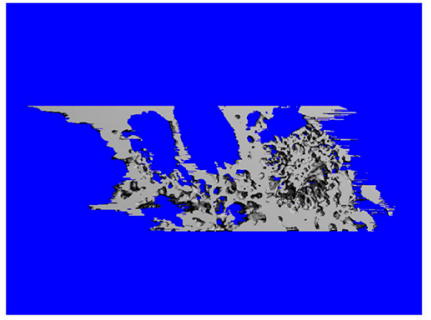

$\mathbf{F}$
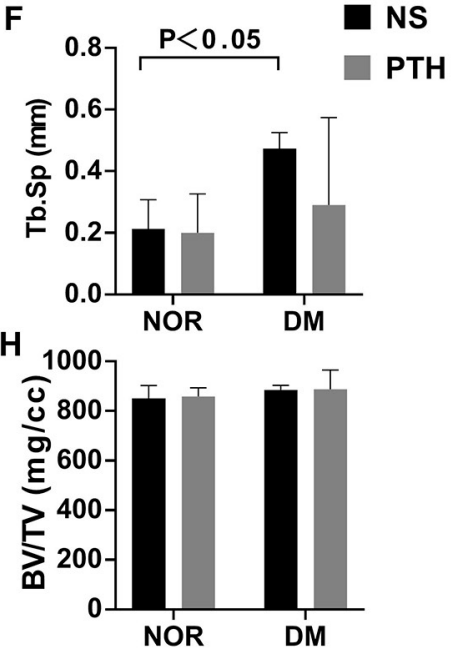

Figure 3- Micro-CT analysis of the extraction socket. (A) Cross section of the Micro-CT scan in the extraction socket; (B) Sagittal plane of the Micro-CT scan in the DM+iPTH group; (C) Sagittal plane of the Micro-CT scan in DM+NS group; (D) 3D reconstruction of the extraction socket in DM+PTH group. (E), (F), (G) and (H) Micro-CT analysis of Tb.N, Tb.Sp, Tb.Th and BMD. Tb.N in NOR+NS group was significantly higher than that in DM+NS group $(p<0.01)$, while the Tb.Sp in NOR+NS group was significantly lower than that in $D M+N S$ group $(p<0.05)$. PTH therapy for 14 days induced no detectable bone anabolic effect in alveolar socket bone parameters 
A

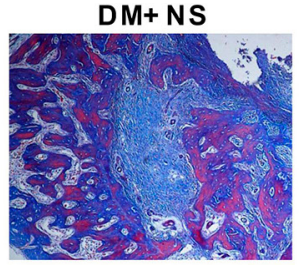

NOR+ NS

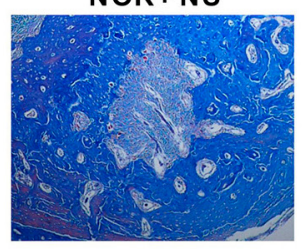

B

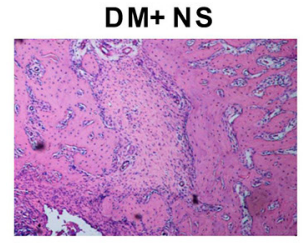

NOR+NS

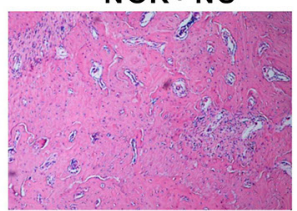

C

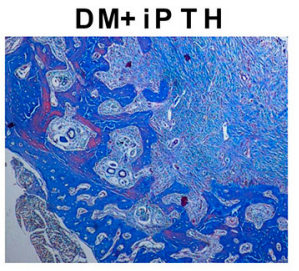

NOR+ iP T H

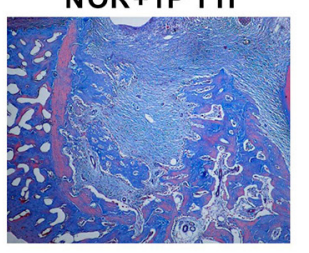

DM+ iP T H

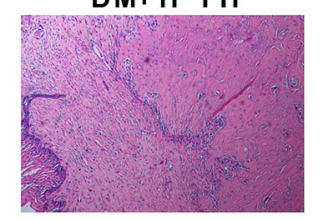

NOR+ IP TH
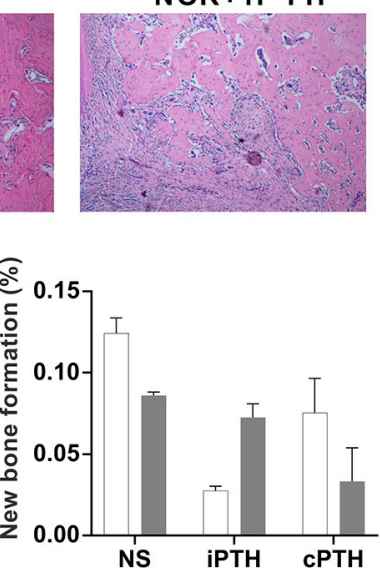

$\mathrm{DM}+\mathrm{c}$ PT H

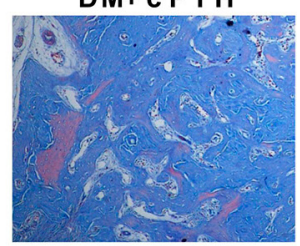

NOR+ c PTH

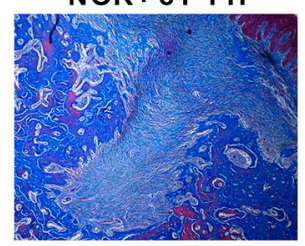

DM+ C P T H

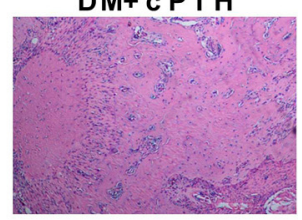

$\mathrm{NOR}+\mathrm{CPTH}$

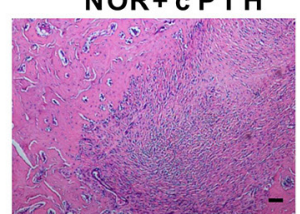

$\square$ NOR

$\square \mathrm{DM}$

Figure 4- Histological analysis of the extraction socket. (A) Masson's trichrome staining (red parts meant new bone and light blue parts meant collagen. Scale bar: $100 \mu \mathrm{m}$ ); (B) HE staining; and (C) New alveolar bone formation in the tooth extraction socket. PTH therapy for 14 days induced no detectable new alveolar bone formation

was found among all the other groups for the alveolar bone Tb.Th and BMD ( $p>0.05$ for all). Bone formation in the extraction socket under the DM conditions in the study was significantly less than that under non-DM condition, indicating an impaired hard-tissue healing of extraction socket under DM condition.

There is no significant difference of Tb.N, Tb.Th, Tb.Sp and BMD between the PTH and NS groups ( $p>0.05$ for all), indicating that the effect of PTH on tooth extraction socket in normal and DM rats was minimal (Figure $2 \mathrm{E}-\mathrm{H}$ ).

\section{Histological analysis of the extraction socket}

The histological analysis of soft tissue (HE and Masson's trichrome staining) showed that more collagen in the extraction socket was observed in the groups treated with PTH (both IPTH and $\mathrm{CPTH}$ ) than those not treated with PTH (Figure 4A and B). The quantitative analysis of hard tissue (new alveolar bone formation) showed no statistical significance between DM and non-DM groups or between NS and PTH groups $(p>0.05)$ (Figure 4C).

\section{Discussion}

In this study, bone formation in the extraction socket in the DM rats was decreased when compared to that of the normal control rats. PTH (1-34) showed no obvious results in hard tissue formation and soft tissue healing. No difference was found on the healing of extraction socket between intermittent and continuous administration of PTH. These findings suggest that hyperglycemic rats failed to benefit from the anabolic treatment of PTH on the healing of extraction socket in the high glucose condition. 
The effectiveness of PTH on promoting the healing of tooth extractions in the diabetic rats was not significant in the study. This may be due to the metabolic characteristics of hyperglycemic rats leading to a condition of ineffective PTH treatment. Some studies showed that PTH did not increase the insulinimmunoreactivity of $\beta$-cells of the pancreatic islets in diabetic rats. ${ }^{24}$ The inflammation caused by diabetes would disrupt vascular endothelial cell function. ${ }^{25}$ The expression of inflammatory cytokines such as TNF-a, IL-1 and IFN-y in bone increased, ${ }^{26,27}$ which contributed to the delayed healing of extraction sockets. Moreover, the high level of advanced glycosylation end products and collagen cross-linking may cause an overall negative effect on diabetic bone mechanics. ${ }^{28}$ Therefore, metabolic control of hyperglycemia may be a key factor when patients with diabetes receive anabolic therapy to improve bone mass. Future studies could explore the anabolic property of PTH in the DM condition when hyperglycemia is controlled with insulin, and immunolabeling or molecular analyses could be performed to further evaluate the possible anabolic activity of PTH and signaling pathways.

The histological analyses in the study showed that the PTH treatment showing more collagen formation demonstrated an anabolic effect on soft tissue healing. In response to hyperglycemia, advanced glycosylation end products were induced and covalently bounded to reactive amino groups, resulting in a dysfunction of the epithelial cells and extracellular matrix. ${ }^{7}$ This is coincident with the decreased collagen in the extraction socket in the DM rats. This may be due to the microcirculation defect and insufficient oxygen caused by thickened and inelastic vessel wall. ${ }^{29}$

Several studies have shown double effects of PTH (1-34) - anabolic when administered intermittently and catabolic when applied continuously. ${ }^{30}$ The subcutaneous and intermittent administration of PTH facilitated the hard and soft tissue repair in tooth extract socket at the dose of $80 \mu \mathrm{g} / \mathrm{kg}$ daily. ${ }^{4}$ Intermittent and systemic PTH administration reduced alveolar bone loss by decreasing the receptor activator of nuclear factor kappa B ligand (RANKL)/ osteoprotegerin (OPG) ratio in hyperglycemic rats. ${ }^{23}$ However, in the continuous administration of $\mathrm{PTH}$, the expression of Phospho1 and Smpd3 in osteoblasts decreased, ${ }^{16}$ resulting in the suppression of bone formation mediated by Cox $2 .^{31}$

Due to the biological nature of hard-tissue healing in the tooth extraction socket, ${ }^{32}$ this study mainly focused on the effects of PTH on bone formation rather than resorption. However, hyperglycemic rats failed to benefit from the anabolic treatment. The contradictory action of PTH might be caused by double effect on bone formation and bone resorption. The anabolic role of iPTH could be lessened by the catabolic effect of DM. ${ }^{33}$ At the early stage of bone and cartilage healing process, both administrations of PTH could promote osteogenic differentiation. ${ }^{34}$ The $\mathrm{CPTH}$ did not suppress osteogenesis, but the catabolic role on bone metabolism was greater than its anabolic role, resulting in bone loss. The $\mathrm{CPTH}$ increased slightly undifferentiated mesenchymal stem cell, osteoblast and osteocyte number at 14 days. ${ }^{35}$ This may result in the non-significant difference between two regimes of PTH. Another possible reason might be that the rats used in the study were male. The cPTH was found to be not catabolic in male rats, as no change was found in BMD and cortical thickness in males. ${ }^{36}$

The experiment period adopted in this study was two weeks. This was in consideration of the optimal slow-release effect of the PECE hydrogel. Furthermore, the epithelialization was generally complete and well-keratinized at approximately two weeks. ${ }^{37}, 38$ The maximum bone formation was achieved and the woven bone completely filled the extraction socket, ${ }^{4}$ and almost replaced by substance with a radio-opacity during that timeframe, ${ }^{37}$ so 14 days was appropriate for this study. The long-term effects of PTH on the healing of extraction socket under hyperglycemic conditions need further investigation.

\section{Conclusion}

Bone formation in the extraction socket under the DM condition was reduced. Hyperglycemic rats failed to benefit from the PTH treatment in the healing of tooth extraction sockets. The different PTH administration regimes (intermittent vs. continuous) had similar effects on the tissues healing.

\section{Acknowledgements}

We thank Professor Shuang Wang from the Department of Geriatrics and Professor Li Zhang from the Department of Hematology, West China Hospital, Sichuan University, China, for the assistance with this study. 


\section{Conflict of interest}

All authors have no conflict of interest.

\section{Funding}

The study was supported by the National Undergraduate Training Program for Innovation and Entrepreneurship of Sichuan University (project No. 201610610379), the Science and Technology Department of Sichuan Province (project No. 2018SC0379) and National Natural Science Foundation of China (No. 81801018)

\section{Authors' Contributions}

Xu, Lin: Conceptualization (Equal); Data curation (Equal); Writing-original draft (Equal); Mei, Li: Conceptualization (Equal); Methodology (Equal); Writing-review \& editing (Equal); Zhao, Rui: Data curation (Equal); Software (Equal); Yi, Jianru: Writingreview \& editing (Equal) Jiang, Yixuan: Data curation (Equal); Software (Equal); Li, Ruomei: Formal analysis (Equal); Resources (Equal); Zhao, Youliang: Data curation (Equal); Pi, Li: Conceptualization (Equal); Methodology (Equal); Software (Equal); Li, Yu: Funding acquisition (Equal); Project administration (Equal); Supervision (Equal); Writing-review \& editing (Equal)

\section{References}

1- International Diabetes Federation. IDF Diabetes Atlas. 9th ed. Brussels: IDF; 2019.

2- Barasch A, Safford MM, Litaker MS, Gilbert GH. Risk factors for oral postoperative infection in patients with diabetes. Spec Care Dentist. 2008;28(4):159-66.

3- Mayard-Pons ML, Rilliard F, Libersa JC, Musset AM, Farge P. Database analysis of a French type 2 diabetic population shows a specific age pattern of tooth extractions and correlates health care utilization. J Diabetes Complications. 2015;29(8):993-7.

4- Kuroshima S, Mecano RB, Tanoue R, Koi K, Yamashita J. Distinctive tooth-extraction socket healing: bisphosphonate versus parathyroid hormone therapy. J Periodontol. 2014;85(1):24-33.

5- Lozano D, Castro L, Dapía S, Andrade-Zapata I, Manzarbeitia F, Alvarez-Arroyo $\mathrm{M}$, et al. Role of parathyroid hormone-related protein in the decreased osteoblast function in diabetes-related osteopenia. Endocrinology. 2009;150(5):2027-35.

6- Zhang $Y$, McClain S, Lee $H$, Elburki M, Yu H, Gu Y, et al. A novel chemically modified curcumin "normalizes" wound-healing in rats with experimentally induced type i diabetes: initial studies. J Diabetes Res [Internet]. 2016 [cited 2020 Mar 2];2016:5782904. Available at: http://dx.doi.org/10.1155/2016/5782904

7- Lioupis C. Effects of diabetes mellitus on wound healing: an update. J Wound Care. 2005;14(2):84-6.
8- Mozzati M, Gallesio G, di Romana S, Bergamasco L, Pol R. Efficacy of plasma-rich growth factor in the healing of postextraction sockets in patients affected by insulin-dependent diabetes mellitus. J Oral Maxillofac Surg. 2014;72(3):456-62.

9- Martínez-Santamaría L, Conti C, Llames S, García E, Retamosa L, Holguín $A$, et al. The regenerative potential of fibroblasts in a new diabetes-induced delayed humanised wound healing model. Exp Dermatol. 2013;22(3):195-201.

10- Al-Obaidi MM, Al-Bayaty FH, Al Batran R, Hussaini J, Khor GH. Impact of ellagic acid in bone formation after tooth extraction: an experimental study on diabetic rats. ScientificWorld]ournal [Internet]. 2014 [cited 2020 Mar 2];2014:908098. Available at: http://dx.doi. org/10.1155/2014/908098

11- Park JJ, Kang KL. Effect of 980-nm GaAlAs diode laser irradiation on healing of extraction sockets in streptozotocin-induced diabetic rats: a pilot study. Lasers Med Sci. 2012;27(1):223-30.

12- Chandu A, Macisaac RJ, Smith AC, Bach LA. Diabetic ketoacidosis secondary to dento-alveolar infection. Int J Oral Maxillofac Surg. 2002;31(1):57-9.

13- Silva BC, Bilezikian JP. Parathyroid hormone: anabolic and catabolic actions on the skeleton. Curr Opin Pharmacol. 2015;22:41-50.

14- Tashjian AH Jr, Chabner B. Commentary on clinical safety of recombinant human parathyroid hormone 1-34 in the treatment of osteoporosis in men and postmenopausal women. J Bone Miner Res. 2002;17(7):1151-61.

15- Keller H, Kneissel M. SOST is a target gene for PTH in bone. Bone. $2005 ; 37(2): 148-58$

16- Houston D, Myers K, MacRae V, Staines K, Farquharson C. The expression of PHOSPHO1, nSMase2 and TNAP is coordinately regulated by continuous PTH exposure in mineralising osteoblast cultures. Calcif Tissue Int. 2016;99(5):510-24

17- Kuroshima S, Kovacic BL, Kozloff KM, McCauley LK, Yamashita J. Intra-oral PTH administration promotes tooth extraction socket healing. J Dent Res. 2013;92(6):553-9.

18- Gou M, Gong C, Zhang J, Wang X, Wang X, Gu Y, et al. Polymeric matrix for drug delivery: honokiol-loaded PCL-PEG-PCL nanoparticles in PEG-PCL-PEG thermosensitive hydrogel. J Biomed Mater Res A. 2010;93(1):219-26.

19- Gong C, Yang B, Qian Z, Zhao X, Wu Q, Qi X, et al. Improving intraperitoneal chemotherapeutic effect and preventing postsurgical adhesions simultaneously with biodegradable micelles. Nanomedicine. 2012;8(6):963-73.

20- Fang Y, Wang LP, Du FL, Liu WJ, Ren GL. Effects of insulin-like growth factor I on alveolar bone remodeling in diabetic rats. J Periodontal Res. 2013;48(2):144-50.

21- Liu M, Zhang J, Wang X. A relevant experimental study of alveolar and systemic bone mineral density changes in diabetes rats. Hua $X$ Kou Qiang Yi Xue Za Zhi. 2009;27(4):451-4.

22- Ersan N, van Ruijven LJ, Bronckers AL, Olgaç V, Ilgüy D, Everts V. Teriparatide and the treatment of bisphosphonate-related osteonecrosis of the jaw: a rat model. Dentomaxillofac Radiol [Internet]. 2014 [cited 2020 Mar 2];43(1):20130144. Available at: http://dx.doi.org/ 10.1259/ dmfr.20130144

23- Chen $\mathrm{H}, \mathrm{Fu} \mathrm{T}, \mathrm{Ma} \mathrm{Y}, \mathrm{Wu} \mathrm{X}$, Li X, Li X, et al. Intermittent administration of parathyroid hormone ameliorated alveolar bone loss in experimental periodontitis in streptozotocin-induced diabetic rats. Arch Oral Biol. 2017;83:76-84.

24- Altan MF, Kanter M, Donmez S, Kartal ME, Buyukbas S. Combination therapy of Nigella sativa and human parathyroid hormone on bone mass, biomechanical behavior and structure in streptozotocin-induced diabetic rats. Acta Histochem. 2007;109(4):304-14.

25- Takahashi S, Kikuchi R, Ambe K, Nakagawa T, Takada S, Ohno T, et al. Lymphangiogenesis and NOS localization in healing process after tooth extraction in akita mouse. Bull Tokyo Dent Coll. 2016;57(3):12131. 
26- Motyl K, Botolin S, Irwin R, Appledorn D, Kadakia T, Amalfitano $A$, et al. Bone inflammation and altered gene expression with type I diabetes early onset. J Cell Physiol. 2009;218(3):575-83.

27- Li YP, Stashenko P. Proinflammatory cytokines tumor necrosis factor-alpha and IL-6, but not IL-1, down-regulate the osteocalcin gene promoter. J Immunol. 1992;148(3):788-94.

28- Campbell GM, Tiwari S, Hofbauer C, Picke AK, Rauner M, Huber $G$, et al. Effects of parathyroid hormone on cortical porosity, nonenzymatic glycation and bone tissue mechanics in rats with type 2 diabetes mellitus. Bone. 2016;82:116-21.

29- Silhi N. Diabetes and wound healing. J Wound Care. 1998;7(1):4751.

30- Silva BC, Costa AG, Cusano NE, Kousteni S, Bilezikian JP. Catabolic and anabolic actions of parathyroid hormone on the skeleton. J Endocrinol Invest. 2011;34(10):801-10.

31- Choudhary S, Canalis E, Estus T, Adams D, Pilbeam C. Cyclooxygenase-2 suppresses the anabolic response to PTH infusion in mice. PloS One [Internet]. 2015 [cited 2020 Mar 2];10(3):e0120164. Available at: http://dx.doi.org/10.1371/journal.pone.0120164 32- Liu GY, Cao GL, Tian FM, Song HP, Yuan LL, Geng LD, et al. Parathyroid hormone (1-34) promotes fracture healing in ovariectomized rats with type 2 diabetes mellitus. Osteoporos Int. 2017;28(10):3043-53.
33- Kuchler U, Spilka T, Baron K, Tangl S, Watzek G, Gruber R. Intermittent parathyroid hormone fails to stimulate osseointegration in diabetic rats. Clin Oral Implants Res. 2011;22(5):518-23.

34- Wolf M, Jäger A, Abuduwali N, Götz W, Lossdörfer S. Continuous PTH modulates alkaline phosphatase activity in human PDL cells via protein kinase $C$ dependent pathways in vitro. Ann Anat. 2013;195(5):455-60. 35- Zhang L, Balani $Y$, Trinh S, Kronenberg $H, M u ~ Y$. [Differential effects on bone and mesenchymal stem cells caused by intermittent and continuous PTH administration]. Zhonghua Yi Xue Za Zhi. 2018;98(10):781-7.

36- Babey M, Wang $Y$, Kubota $T$, Fong $C$, Menendez A, ElAlieh $H$, et al. Gender-specific differences in the skeletal response to continuous pth in mice lacking the IGF1 receptor in mature osteoblasts. J Bone Miner Res. 2015;30(6):1064-76.

37- Smith N. A comparative histological and radiographic study of extraction socket healing in the rat. Aust Dent J. 1974;19(4):250-4. 38- Yi J, Mei L, Li X, Zheng W, Li Y, Zhao Z. Effects of continuous and intermittent parathyroid hormone administration on midpalatal suture expansion in rats. Arch Oral Biol. 2019;99:161-8. 